\title{
DOUBLE-BLIND EVALUATION OF THE EFFICACY AND SAFETY OF TEMAZEPAM IN OUTPATIENTS WITH INSOMNIA
}

\author{
J.M. FILLINGIM \\ Chief of Staff, Candler General Hospital, Savannah, Georgia
}

1 The efficacy and safety of temazepam $30 \mathrm{mg}$, compared with glutethimide $500 \mathrm{mg}$ and placebo, were evaluated in double-blind conditions in a 4-day study in 75 outpatients with a history of insomnia. 2 Temazepam and glutethimide were rated by the patients as effective and significantly superior to placebo for general quality of sleep, time required to fall asleep, frequency of nocturnal and early morning awakenings, and duration of sleep.

3 Residual effects reported for temazepam and glutethimide immediately after awakening and during the day were similar to or less than those reported for placebo.

\section{Introduction}

DRUG therapy for insomnia includes the 'shortacting' barbiturates (pentobarbitone, quinalbarbitone, and so on), the glutarimides (glutethimide and methyprylon) and the benzodiazepines (principally flurazepam and nitrazepam). All of these initially improve insomnia when administered in appropriate doses. However, barbiturates have several disadvantages, including the onset, within several days, of sleep stage abnormalities, and tolerance and withdrawal phenomena; even single doses have been reported to produce depressant effects which persist into the following day. The glutarimides and methaqualone, which resemble the barbiturates chemically and pharmacologically, have similar disadvantages. Moreover, because of their high solubility in lipids, an overdosage produces symptoms which are particularly difficult to treat (Jinks, 1976; Drug and Therapeutics Bulletin, 1971; Medical Letter, 1970).

\section{Methods}

Seventy-five men and women between the ages of 18 and 59 were enrolled in the study. They had a documented history of chronic or sporadic insomnia characterized by: (a) either difficulty in falling asleep, involving a sleep-induction time of more than $30 \mathrm{~min}$, but preferably more than $60 \mathrm{~min}$, or one or more awakenings during the night with difficulty in returning to sleep without a known cause for awakening, for example, pain; and (b) less than $6 \mathrm{~h}$ sleep per night followed by tiredness in the morning. Further, each patient had experienced insomnia for a minimum of 10 weeks during the preceding year, and his individual episode of insomnia had a usual duration of 2 weeks.

Patients with any mental disability which could interfere with proper completion of the study questionnaire were excluded from the trial, as were those with a history of gastrointestinal, liver or kidney dysfunction, unstabilized cardiovascular disease, chronic clinical illness, drug addiction or hypersensitivity to drugs chemically similar to the test medications. Pregnant or lactating women were excluded, as were patients who had taken another investigational drug within 4 weeks of the start of the study, or who had received, within the past 3 months, any drug with a well-defined potential toxicity.

All patients selected for the study had used a chemotherapeutic agent for their sleep disturbances in the past; however, an adequate washout period for these drugs was observed before starting the study drug. Any medication similar to either of the active agents being tested, such as other hypnotics, sedatives, analgesics and tranquilizers, were forbidden during the study, but other necessary medications were permitted. Women of child-bearing age were required to use a medically approved method of birth control.

Patients were randomly assigned to one of the three treatment groups: temazepam $30 \mathrm{mg}$, glutethimide $500 \mathrm{mg}$ or placebo. The medications were provided in identical hard gelatin capsules to comply with the double-blind study design.

Each patient was given four capsules of his medication, with instructions to take one capsule each night immediately before retiring for 4 consecutive nights. In addition, the patient was asked to take 
the capsules at the same time each night, that is, at approximately 23.00 , if possible, and to refrain from drinking any tea, coffee or alcohol after the evening meal.

\section{Evaluation of efficacy}

Efficacy evaluations were based on (a) the patients' assessment of the drug's effect on several features of associated sleep disturbances and (b) the physicians' global evaluation of the effect of the drug on the patients' insomnia.

Each patient completed a Daily Sleep Questionnaire designed to obtain qualitative information on his or her sleep patterns while taking the study medications. The specific variables evaluated were (1) time to fall asleep, (2) nocturnal awakening(s), (3) early morning awakenings, (4) general quality of sleep and (5) general effectiveness of the study medication compared with sleep preparations used previously. To indicate duration of sleep, the patient recorded how long it took to fall asleep, as well as the exact time he went to bed and awoke the following morning. In addition, the patient noted any residual effects of the medication immediately after awakening and during the day.

These evaluations were made following each of the 4 consecutive nights that the drug was taken. For purposes of statistical analysis, the patients' qualitative responses were assigned scoring values of 1-7, where 1 represented the maximum response and 7 the worst.

The physicians' evaluation was a qualitative assessment, at the end of the treatment, of the overall therapeutic effectiveness of the study medications based on a comparison of the study drugs with (a) no medication and (b) the most effective previous medication. The qualitative responses were assigned values of 1-5, where 1 represented the best possible result and 5 the worst.

\section{Evaluation of safety}

During the week before therapy, and on the final day of the study, each patient received a complete ophthalmological and physical examination, including vital signs (blood pressures, radial pulse rates and temperature). In addition, laboratory tests, including urinalysis, blood chemistries, haemoglobin, haematocrit, and a complete blood count with platelet estimate, were carried out. Each patient also recorded daily in the Questionnaire any unusual feelings while on the study drug. At the final visit the patients' comments were reviewed, and each was questioned regarding any possible adverse effects noted during the study, the incidents, types, severity and duration of which were reported by the physician. The severities of the adverse drug reactions were rated using scoring values of $1=$ mild, $\mathbf{2}=$ moderate and $\mathbf{3}=$ severe.

\section{Statistical procedures}

Two statistical procedures were used to analyse the efficacy data. For the variables in the patients' Daily Sleep Questionnaire, a repeated measurements model of analysis was applied to the ratings made on each day following each night of medication to detect any differences between the effects of temazepam or glutethimide, compared with those of placebo, on each of the 4 consecutive study days, and on the 4 study days combined (that is average of all evaluations).

A contingency table analysis was applied to the physician's global assessments to determine any possible differences in the frequency distribution of patients in each treatment group who were much better, a little better, no different, a little worse, or much worse after each night on medication, and throughout the study (average score).

Analysis of variance was used to evaluate significant differences between the treatment groups in the analysis of the patients' self-evaluation questions, the $\chi^{2}$ test for independence was applied to detect any significant between-group differences in the contingency tables. Pair comparisons between the treatment groups were made using the least significant difference test.

\section{Results}

Seventy-five patients (17 males and 58 females; age range 18-59 yr) were chosen for this study. The three study drugs were evenly distributed among the 75 patients, with one exception. One patient receiving temazepam could not remember how helpful the previous medication had been; and so the data on temazepam were based on 24 patients. The patients in the three groups were similar with respect to age, sex, race, height and weight. The majority of the patients suffered from sporadic $(50 \%)$ or chronic $(43 \%)$ insomnia, whereas only $7 \%$ had acute insomnia. The severity was considered moderate in $74 \%$ of the patients, mild in $19 \%$ and severe in $7 \%$. Typically, the patients' sleep disturbances had begun about $5.7 \mathrm{yr}$ before, and affected them approximately 37 weeks per year. For most patients, the usual duration of each period of insomnia was between 1-13 weeks.

Approximately $96 \%$ of the patients complained of difficulty in falling asleep, with a mean sleep induction time of 96 minutes. Most $(96 \%)$ of the patients also reported that they awakened frequently in the night; in fact, $68 \%$ commonly awoke two or three times per night. All but one patient reported 
early morning awakenings. Because the patients slept only $4.3 \mathrm{~h}$ (mean value) on a typical night, as might be expected, all complained of insufficient sleep resulting in a tired feeling the following morning.

In compliance with the protocol, all patients had taken medication for their sleep disturbances before entering the study. Review of the medical histories revealed that 26 different drugs had been taken. Of the 26 , the three most frequently used medications and the percentages of patients using them were: diazepam (Valium ${ }^{\circledast}$, Roche) $56 \%$, clorazepate dipotassium (Tranxene ${ }^{\oplus}$, Abbott) $25 \%$ and flurazepam hydrochloride (Dalmane ${ }^{\circledR}$, Roche) $20 \%$. All patients reported good to excellent effectiveness of these drugs on their sleep disturbances, with the exception of one individual who reported only fair improvement while on clorazepate dipotassium.

\section{Evaluation of efficacy}

Table 1 summarizes the results of the analysis of the patients' responses for the variables which reflect the common complaints of patients with sleep disturbances. As difficulty falling asleep is generally the most common complaint, the drugs' effects on "sleep induction" were assessed by asking the patient two questions: "How long did it take you to fall asleep last night as compared to when you took no sleep medication?" and "Estimate the time (in minutes) that it took you to fall asleep".

Sleep induction on the 4 consecutive nights of the study, both individually and combined, was improved by temazepam, glutethimide and, to a lesser extent, by placebo (Table 1). Between-group comparisons revealed that temazepam and glutethimide were about equal to each other, but significantly better than placebo in reducing the time required to fall asleep on the four nights combined and on nights 2,3 and 4 individually.

These findings were consistent with the mean values for the patients' estimates of time required to fall asleep. For nights 1-4 the mean values were 39 min with temazepam, 43 min with glutethimide and 73 min with placebo. Thus, the group treated with temazepam fell asleep twice as fast as the group receiving placebo overall during the study. These mean values also showed that less time was required to fall asleep on each night of the study by the patients treated with either temazepam or glutethimide than by those treated with placebo; each active drug was favoured significantly over placebo for nights 1,2 and 4.

Staying asleep is another problem patients with insomnia experience. Therefore, the drugs' effects on

Table 1 Summary of patient self-evaluation analyses: effects of study medications on sleep disturbances

\begin{tabular}{|c|c|c|c|c|c|c|c|}
\hline Parameter & Treatment & $\begin{array}{l}\text { No. of } \\
\text { points }\end{array}$ & 1 & $\begin{array}{l}\text { Stu } \\
2\end{array}$ & Mean valuest & 4 & $\begin{array}{c}\text { Overall } \\
\text { nights } 1-4\end{array}$ \\
\hline Sleep induction & $\begin{array}{l}\text { Temazepam } \\
\text { Glutethimide } \\
\text { Placebo }\end{array}$ & $\begin{array}{l}25 \\
25 \\
25\end{array}$ & $\begin{array}{l}2.56 \\
2.72 \\
3.24\end{array}$ & $\begin{array}{l}2.63 \longrightarrow * \\
2.44 * * \\
3.60 \doteq\end{array}$ & $\left.\begin{array}{l}2.12 \\
2.32 \\
3.12\end{array}\right]$ & $\begin{array}{l}2.36 \\
2.127^{*} \\
3.64\end{array}$ & $\begin{array}{l}2.43 \\
2.40 * * \\
3.40 \doteq\end{array}$ \\
\hline $\begin{array}{l}\text { Sleep induction } \\
\text { time }(\min )\end{array}$ & $\begin{array}{l}\text { Temazepam } \\
\text { Glutethimide } \\
\text { Placebo }\end{array}$ & $\begin{array}{l}25 \\
25 \\
25\end{array}$ & $\left.\begin{array}{l}41 \\
537 \\
80\end{array}\right]$ & $\begin{array}{l}41 \square \\
437 \\
75 \beth\end{array}$ & $\begin{array}{l}35 \\
40 \\
57\end{array}$ & $\begin{array}{l}37-{ }^{*} \\
36 * * \\
77 \beth\end{array}$ & $\begin{array}{l}39 \square \\
437 \\
73 \pm\end{array}$ \\
\hline $\begin{array}{l}\text { Nocturnal } \\
\text { awakenings }\end{array}$ & $\begin{array}{l}\text { Temazepam } \\
\text { Glutethimide } \\
\text { Placebo }\end{array}$ & $\begin{array}{l}25 \\
25 \\
25\end{array}$ & 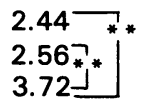 & $\left.\begin{array}{l}2.40 \\
2.04 * \\
3.64\end{array}\right]^{*}$ & $\begin{array}{l}2.12 \square \\
2.20 \exists \\
3.16 \exists\end{array}$ & $\begin{array}{l}2.04 \longrightarrow * \\
1.68 * * \\
3.24 \exists\end{array}$ & $\begin{array}{l}2.25-* \\
2.12 * * \\
3.44=1\end{array}$ \\
\hline $\begin{array}{l}\text { Early morning } \\
\text { awakenings }\end{array}$ & $\begin{array}{l}\text { Temazepam } \\
\text { Glutethimide } \\
\text { Placebo }\end{array}$ & $\begin{array}{l}25 \\
25 \\
25\end{array}$ & $\begin{array}{l}1.88 \\
1.92 \\
2.64\end{array}$ & $\begin{array}{l}2.12 \sqsupset{ }^{*} \\
2.16 \neg{ }^{3.20}\end{array}$ & $\begin{array}{l}2.04 \\
2.08 \\
2.76\end{array}$ & $\begin{array}{l}1.80 \\
1.64 \neg * \\
2.80 \beth^{*}\end{array}$ & $\left.\begin{array}{l}1.96 \\
1.95 \\
2.85\end{array}\right]$ \\
\hline $\begin{array}{l}\text { Duration of } \\
\text { sleep (min) }\end{array}$ & $\begin{array}{l}\text { Temazepam } \\
\text { Glutethimide } \\
\text { Placebo }\end{array}$ & $\begin{array}{l}25 \\
25 \\
25\end{array}$ & $\begin{array}{l}394 \\
412 \\
376\end{array}$ & $\begin{array}{l}427 \\
416 \\
378\end{array}$ & $\left.\begin{array}{l}436 \\
418 \\
383\end{array}\right]$ & $\begin{array}{l}432 \square \\
437 \beth^{*} \\
379\lrcorner\end{array}$ & $\begin{array}{l}422 \square * \\
421 \neg{ }^{*} \\
379\lrcorner\end{array}$ \\
\hline $\begin{array}{l}\text { Quality of } \\
\text { sleep }\end{array}$ & $\begin{array}{l}\text { Temazepam } \\
\text { Glutethimide } \\
\text { Placebo }\end{array}$ & $\begin{array}{l}25 \\
25 \\
25\end{array}$ & $\begin{array}{l}2.24 \\
2.20 ユ_{*}^{*} \\
3.48 A^{*}\end{array}$ & $\begin{array}{l}2.48 \square \\
1.84 * * \\
3.36\lrcorner\end{array}$ & $\begin{array}{l}2.16 \\
2.12 \beth^{*} \\
3.44 \beth\end{array}$ & $\begin{array}{l}2.00-7^{*} \\
1.96 * * \\
3.56 \doteq\end{array}$ & $\begin{array}{l}2.22 \vec{*}^{*} \\
2.03 * * \\
3.46=\end{array}$ \\
\hline
\end{tabular}

Significance levels: $* P<0.05 ; * *<0.01$.

† For qualitatively ordered variables, a mean value of 4.00 indicates the same effect as when no sleep medication was taken; the lower the mean value, the greater the improvement reported. 
the parameter "nocturnal awakenings", a measurement of sleep maintenance, were evaluated on the basis of the responses by the patients to the question, "How often did you wake up last night after you fell asleep as compared to when you took no sleep medication?"'.

The results presented in Table 1 show that temazepam and glutethimide were about equally effective in reducing nocturnal awakenings (a) throughout the study (that is, nights 1-4), as well as (b) on each of the 4 consecutive study nights. Further, each of the active drugs was significantly more effective than placebo in lessening nocturnal awakenings.

Another common problem for patients with sleepmaintenance difficulties is the tendency to awaken very early in the morning. To evaluate the drugs' effects on the parameter, "early morning awakening", the patients were asked "Did you wake up earlier in the morning than you had to and could not fall back to sleep again? (Compare with when you did not take any sleep medicine)."

Analyses of the patients' responses to this question revealed that temazepam and glutethimide were equally effective in decreasing the patients' problem of early morning awakening, and that treatment with either of these drugs was significantly more effective than placebo treatment over the four nights considered together and on the individual nights 2 and 4.

The tendency to awaken early in the morning obviously interferes with the patients' desired "duration of sleep". The length of sleep is an important feature, as it is a critical factor in maintaining good health. In the study, this variable was calculated as the interval between the time the patient reported awakening in the morning and the time he or she went to bed the night before, minus the estimated time taken to fall asleep.

The mean values for duration of sleep presented in Table 1 indicate that patients given the active drugs slept longer than did patients receiving placebo (that is, 43 min longer with temazepam and $\mathbf{4 2} \mathrm{min}$ longer with glutethimide). This represents an $11 \%$ increase in length of sleep throughout the study for the patients treated with the active drugs.

Temazepam and glutethimide were each significantly superior to placebo in extending the duration of sleep throughout the study. In addition, temazepam was significantly superior to placebo in increasing the length of sleep on nights 3 and 4; glutethimide, however, was significantly superior to placebo only on night 4 .

Although individual sleep parameters, such as sleep induction, nocturnal and early morning awakenings and duration of sleep, may be improved, it is essential that the patient with insomnia feels he has obtained a good night's sleep overall. To evaluate the effectiveness of the drugs in improving the "quality of sleep" the patients were asked, "How well did you sleep last night as compared to when you took no sleep medicine?"

The results (Table 1) demonstrate that both temazepam and glutethimide improved the quality of sleep throughout the study. Furthermore, each drug was significantly superior to placebo in improving the quality of sleep on the four individual study nights, as well as over all these nights.

\section{Residual effects}

To assess any residual effects the patients were asked "How awake do you feel this morning as compared to when you took no sleep medicine?" and "How alert did you feel today as compared to when you took no sleep medicine?". The first question was to be answered immediately on arising, and the second just before the evening meal. Neither temazepam nor glutethimide caused any residual effects the morning after treatment.

\section{Relative effectiveness}

In general, the statistical analyses emphasized the effects of the study medications on the key complaints of patients with sleep disturbances. However, it was also considered essential to evaluate the effectiveness of each of the three agents relative to previous sleep medications used by the patient. Therefore, the patients were asked, "In general, is this medication better or not better than the medicine you took before to help you sleep?". Patients who had received either temazepam or glutethimide considered these medications to be significantly more helpful than medications used previously for their sleep disturbances compared with patients who took placebo throughout the study.

In the comparison of the effectiveness of the study treatments with that of "no previous medication", in 21 out of 25 patients in each of the two active drug groups, temazepam or glutethimide were considered better than no treatment whatsoever. Consistent with this was the result that in $\mathbf{1 2}$ out of 25 patients given placebo, treatment effectiveness was considered as "no different" from when the patients took no medication.

When the effectiveness of the study treatments was compared with that of the most effective previous medication, in 19 out of the 25 patients receiving either temazepam or glutethimide, the effectiveness of these two treatments was judged better than that of the most effective previous medication. On the other hand, 15 out of the 25 patients given placebo rated it as less effective (that is, "worse") than the most effective previous medication. 


\section{Evaluation of safety}

No changes were noted during the final physical and ophthalmological examinations attributable to the test medications. No clinically or statistically significant changes in the patients' oral temperatures, respiratory rates, systolic and diastolic blood pressures (supine and standing), or radial pulse rates (supine and standing) were found, with the exception of a significant increase of $7.0 \mathrm{~mm} \mathrm{Hg}$ standing systolic blood pressure from baseline in the group treated with glutethimide.

All pre- and post-treatment clinical laboratory values were within ranges acceptable for the safety and efficacy requirements of this study.

\section{Adverse reactions}

A total of 16 different adverse reactions considered to be either definitely or possibly drug-related were reported by four patients receiving temazepam, six receiving glutethimide and seven receiving placebo. The reactions were mild or moderate with the exception of severe dry mouth and euphoria reported by one patient after the first night of treatment with glutethimide. However, in no instance was the adverse reaction severe enough to necessitate discontinuation of therapy.

\section{Discussion}

The primary goal of the present study was to compare the effectiveness of temazepam with that of glutethimide and placebo. The evaluation, completed in double-blind conditions, was based on the patients' self-appraisals of drug effects. This approach, in contrast with that of the sleep laboratory, permitted the testing of the drugs in a large number of patients in their homes. The second goal of this study was to evaluate any residual effects of the drugs.

The patients' evaluation showed that temazepam and glutethimide were considered overall significantly superior to placebo; they were rated as superior to placebo for the residual effects after awakening and during the day. Differences favouring temazepam and glutethimide over placebo appeared after only one dose. Several interesting comparisons can be made between temazepam and glutethimide. Both were equally effective, and patients receiving either of the active drugs estimated that they fell asleep in about one-half the time of patients on placebo.

Patients receiving either temazepam or glutethimide also awoke less frequently during the night, with fewer disturbances of early morning sleep. The duration of sleep was prolonged by almost $45 \mathrm{~min}$ in patients receiving either of the two active drugs, as compared with the sleeping time of placebo patients.

Residual effects after temazepam or glutethimide were no greater than with placebo. In fact, patients after having taken temazepam or glutethimide consistently reported being more alert both in the morning and for the entire day following treatment than did the placebo group, a finding which reinforced the patients' claims of improved quality of sleep.

The efficacy of both temazepam and glutethimide was further supported by the evaluations, by both the patients and the physician-investigator, of the relative effectiveness of the study treatments compared with sleep medications used by the patients before the study. Placebo, in contrast, tended to be less effective than the previously used sleep medications, indicating that the patients could indeed reliably discriminate between the effects of the active drugs and placebo, thereby further supporting the efficacy of temazepam and glutethimide. Moreover, the physician's global assessment indicated that temazepam and glutethimide were more effective than no treatment whatsoever, and placebo was no different from no treatment.

Based on these evaluations, temazepam was judged to be a safe and efficacious drug for the treatment of sleep disturbances.

\section{References}

JINKS, M. (1976). Insomnia and its treatment. J. Am. Pharm. Ass., NS16, 613-616.

Sleeping pills: the day after (1971). Drug Ther. Bull., 9, (3), 9-10.

Flurazepam (Dalmane), a new hypnotic (1970). Med. Lett., 12, (22), 89-90. 